\title{
ÁCIDOS GRASOS OMEGA-3 Y CÁNCER, UNA ALTERNATIVA NUTRICIONAL PARA SU PREVENCIÓN Y TRATAMIENTO
}

\section{OMEGA-3 FATTY ACIDS AND CANCER, A NUTRITIONAL ALTERNATIVE FOR ITS PREVENTION AND TREATMENT}

\author{
Rodrigo Valenzuela B. (1), Karla Bascuñan G.(1), \\ Rodrigo Chamorro M.(2), Alfonso Valenzuela B. (3). \\ (1) Escuela de Nutrición y Dietética, Facultad de Medicina, \\ Universidad de Chile. Santiago, Chile. \\ (2) Laboratorio de Sueño, Instituto de Nutrición y Tecnología de los Alimentos (INTA) \\ Universidad de Chile. Santiago, Chile.
}

(3) Centro de Lípidos. Instituto de Nutrición y Tecnología de los Alimentos (INTA), Universidad de Chile y Facultad de Medicina, Universidad de los Andes. Santiago, Chile.

\begin{abstract}
Long-chain omega-3 polyunsaturated fatty acids (omega-3 LCPUFA) are essential for human beings and they should be incorporated to our body through the diet. For almost a century it is known that these fatty acids are essential for growth and development of individuals and from 1970 onwards, investigations have found solid evidence about several health properties attributed to these fatty acids, especially at the cardiovascular, neurological and anti-inflammatory level; it is in this sense that today a large number of investigations have focused to study the protective role of omega-3 LCPUFA against cancer, primarily based on the anti-inflammatory properties that they possess. In this paper we describe a series of evidences to establish a possible preventive and therapeutic use of omega-3 LCPUFA against cancer, specifically in prostate, breast and colon cancer.
\end{abstract}

Key words: cancer, fatty acids, omega-3 fatty acids, anti-inflammatory effect.

Este trabajo fue recibido el 13 de Diciembre de 2010 y aceptado para ser publicado el 10 de Mayo de 2011.

\section{INTRODUCCIÓN}

En los países occidentales, las enfermedades cardiovasculares (ECV), el cáncer $(\mathrm{Ca})$, la obesidad $(\mathrm{Ob})$ y la diabetes tipo 2 (DM2), son responsables de más del $80 \%$ de la mortalidad (1). Se ha propuesto que los lípidos desempeñarían un papel decisivo en el desarrollo de éstas enfermedades, particularmente en referencia a la cantidad y tipo de ácidos grasos (AG) aportados por la dieta.

La ingesta total de lípidos, así como la relación en el consumo de ácidos grasos poliinsaturados (AGPI) de cadena larga (AGPICL) omega-6/omega-3, ha aumentado considerablemente en la dieta occidental en las últimas décadas (2). Es ya conocido que la dieta estándar actual difiere sustancialmente de aquella que consumieron los seres humanos desde tiempos prehistóricos (3). Por otro lado, está relativamente bien documentado el efecto preventivo de los AGPICL omega-3 en las ECV, ciertos
Ca, DM2 y en el síndrome metabólico (SM), a través de información derivada tanto de estudios clínicos como epidemiológicos $(4,5)$.

Si bien el efecto del medio ambiente en la expresión de los genes es aún controversial, existe certeza que la dieta puede modificar la expresión de nuestro patrimonio genético, afectando tanto en forma positiva, como también negativa, en la salud de los seres humanos (6). Es más, en relación a nutrientes como los lípidos, se sabe que la cantidad y el tipo específico de estos en la dieta, puede tener un impacto marcado y diferencial en la salud de animales con la misma base genética, incluyendo los humanos, así como en la salud de poblaciones predispuestas genéticamente (7 - 9). En el presente trabajo se expone una visión general sobre los beneficios de los AGPICL omega-3 de origen dietario, en relación a su posible efecto preventivo en el desarrollo de ciertos $\mathrm{Ca}$, 
como el cáncer de próstata $(\mathrm{CaP})$, el cáncer de mama $(\mathrm{CaM})$, y el cáncer de colon $(\mathrm{CaC})$. La información deriva de las evidencias clínicas y epidemiológicas disponibles en relación a estas patologías de alta prevalencia actual en la población occidental, cuya alimentación se caracteriza por una baja ingesta de AGPICL omega-3 y de una distorsionada relación entre el consumo de estos ácidos grasos y aquellos de la serie omega-6.

\section{APORTE DIETARIO Y METABOLISMO DE LOS AGPICL Fuentes alimentarias de AGPI omega-3 y omega-6}

El ser humano sintetiza a partir de novo los AG saturados y monoinsaturados. Sin embargo, carece de la maquinaria enzimática para sintetizar AGPI, por lo que estos deben ser aportados por la dieta, por lo cual son denominados ácidos grasos esenciales (AGE). (10) Estos son los AGPI omega-3 y omega-6 y sus respectivos AGPICL derivados de ellos. Los vegetales terrestres, especialmente aquellos con alto contenido de lípidos, pueden sintetizar el primer precursor de los AGPICL omega-6, el ácido linoleico (C18:2, $\Delta$ 9, 12; omega-6; AL). Es así como los aceites de maravilla (girasol), de soja, pepa de uva y de maíz, entre otros, constituyen una buena fuente dietaria de AL. Por otro lado, el primer precursor de los AGPICL omega-3, el ácido alfa linolénico (C18:3, $\Delta$ 9, 12, 15; omega-3; ALN), es más abundante en los aceites de raps (canola), nuez, linaza y chía, entre otros, y en menor proporción en el aceite de soya (uno de los aceites más consumidos en occidente) (11). Los AGPICL omega-3 de mayor importancia son el ácido eicosapentaenoico (C20:5, $\Delta 5,8,11,14,17$; EPA) y el ácido docosahexaenoico (C22:6, $\Delta 4,7,10,13,16,19$; DHA), los que sólo están presentes en los vegetales y animales de origen marino, siendo estos últimos nuestra principal fuente nutricional, particularmente los peces de aguas frías (salmónidos, jurel, sardina, caballa) o profundas (atún, bacalao) (12) quienes los obtienen del fito y zooplancton que forma parte de su cadena alimentaria (13). La población occidental, lamentablemente consume pocos vegetales de origen marino, los que son particularmente ricos en DHA (14). Es importante señalar que el contenido de AGPICL omega-3 de los peces varía considerablemente en función de la especie, contenido de materia grasa y ubicación geográfica de estos. Los peces de aguas profundas y de temperaturas frías tienen, un mayor contenido de EPA y DHA que los peces de cultivo (salmónidos), ya que estos pueden tener una variación significativa en sus niveles de AGPICL omega-3, derivado principalmente de las características de la dieta artificial que se suministra a estos peces (15).

\section{Conversión de AL y de ALN en AGPICL}

Los mamíferos, con la excepción de los felinos que no pueden sintetizar de novo AGPICL omega-3 y omega- 6 , pueden convertir al AL y al ALN en sus derivados de mayor tamaño de cadena y poliinsaturación; ácido araquidónico (C20:4 $\Delta$ 5, 8, 11, 14; omega-6, AA) en el caso del AL y EPA y DHA, en el caso del ALN, dado que en sus células se encuentran las enzimas elongasas y desaturasas necesarias para su biosíntesis. Estas enzimas aumentan el tamaño de la cadena de carbonos e introducen nuevos dobles enlaces, respectivamente, a los precursores AL y ALN (16). La conversión ocurre, principalmente, en el retículo endoplasmático liso celular en una primera etapa y en el caso específico de los AGPICL omega-3, continúa en los peroxisomas (17).

\section{EFECTOS PROTECTORES DE LOS AGPICL OMEGA-3 FRENTE AL CÁNCER}

Estudios de diseño caso-control, realizados en Japón (18) y en Escocia (19) han demostrado el papel protector de AGPICL omega-3 frente al $\mathrm{Ca}$, al observar una relación inversa entre el riesgo de desarrollar esta patología y el mayor consumo de AGPICL omega-3, siendo esta asociación fuertemente significativa para el $\mathrm{CaC}$ distal (20). Por otro lado, un estudio en el que se midieron los niveles de AG en sangre, estableció una relación inversa entre la concentración total de AGPICL omega-3 y el riesgo de desarrollar CaP (21). En un estudio realizado en Jamaica, se observó que hombres sometidos a biopsia de próstata por elevación en los niveles del antígeno prostático específico, presentaban una correlación positiva entre los niveles de ALN y de DHA en los fosfolípidos de membrana de los eritrocitos y un menor volumen tumoral en la próstata (22).

Los antecedentes epidemiológicos actualmente disponibles permiten establecer una asociación entre la ingesta de AGPICL omega-3 y el cáncer, a través de estudios de correlación que sugieren un efecto protector de estos AG (23). Sin embargo, no todos los resultados son totalmente concluyentes, tema que aún requiere más estudios (24). Esta discordancia se podría explicar por variaciones tanto en la cantidad como de la fuente utilizada de AGPI omega-3 (de origen terrestre, ALN, o marino, EPA y/o DHA). Muchos estudios se basan en datos de ingesta dietética (encuestas de libre consumo) o de estimaciones basadas en consumos nacionales, con lo cual estas evaluaciones se correlacionan pobremente con mediciones directas de los AG en los individuos. En algunos de ellos, la ingesta de AGPICL omega-3 pudo ser demasiado baja para observar un efecto protector (algo frecuente en la población occidental), o el efecto protector pudo ser mitigado por contaminantes u otros 
componentes alimentarios.

Otro aspecto a considerar es que la relación de consumo omega-6/omega-3 puede ser más importante que la ingesta absoluta de AGPICL. En un estudio realizado en ratones knockout Pten (animales inmuno competentes, predispuestos genéticamente a desarrollar $\mathrm{CaP}$ ) en los cuales se evaluaron indicadores prostáticos específicos, se estableció que cuando existe una relación de consumo omega-6/omega-3 inferior a cinco, se produce una disminución significativa de la progresión del CaP (25).

Considerando el caso de los humanos, es posible esperar que distintas poblaciones consuman diferentes cantidades de AGPI omega-6 y omega-3, pudiendo existir diferencias genéticas o epigenéticas en la actividad de las enzimas responsables de las biotransformaciones de estos AG a sus respectivos derivados de cadena larga. Por lo cual, es necesario individualizar la cantidad ingerida de AGPI o AGPICL omega-6 y omega-3, con el fin de poder identificar una relación eficaz entre estos AG. También es posible que los resultados dependan de la forma en que consumen o administran estos AG, esto es en la forma de triglicéridos, fosfolípidos, libres, esterificados, entre otros (26) ya que hay antecedentes que establecen diferencias en la biodisponibilidad de los AG según la estructura que los contiene, particularmente en el caso de los AGPICL omega-3 (27). Aún no está claro si los diferentes tipos de AGPI omega-3 consumidos o utilizados en los estudios, son igualmente eficaces en la supresión de tumores, por lo que resulta clave, en futuros estudios, considerar la real tasa de conversión de ALN en EPA y/o DHA. Por último, los efectos de los AGPICL omega-3, tanto en el riesgo de desarrollar un cáncer, como en la evolución de este, pueden estar influenciados por factores genéticos, tales como los polimorfismos que podrían producir una expresión y una respuesta distinta frente a la exposición a AGPI o AGPICL omega-3 (28). Algunos estudios, por ejemplo, indican una posible interacción entre los AGPICL omega-3 dietarios y polimorfismos en la expresión de las isoformas de las enzimas ciclooxigenasas (COX) y lipoxigenasas (LOX), así como en la expresión de ciertos genes que podrían determinar el riesgo de presentar un cáncer (29).

\section{USO DE LOS AGPICL OMEGA-3 EN MODELOS ANIMALES Y CELULARES ESPECÍFICOS DE CÁNCER}

Las dificultades prácticas para llevar a cabo estudios de intervención en humanos respecto a la función protectora de los AGPICL omega-3 frente al cáncer, obligan a realizar estos estudios en animales y en líneas celulares específicas. A continuación se presentan antecedentes que demuestran el efecto protector de los AGPICL omega-3, frente a patologías específicas como el CaP, el CaM y el CaC.

\section{Modelos de estudio de cáncer prostático $(\mathrm{CaP})$}

Los primeros estudios que demostraron un efecto inhibitorio significativo en el crecimiento de células cancerosas humanas de origen prostático injertadas en ratones, utilizaron aceite de pescado cuyo contenido de AGPICL omega-3 es relativamente alto (25-30\%) (30). Más recientemente, un estudio que también utilizó injertos de células cancerosas humanas en ratones que tenían una inmunodeficiencia grave, demostró una reducción significativa en el crecimiento y el volumen del tumor y en los niveles séricos del antígeno prostático específico, al utilizar suplementos nutricionales con AGPICL omega-3 (31). En estos animales se produjo una reducción significativa en el crecimiento tumoral, un retraso en la progresión histopatológica del tumor y un aumento en la sobrevida de estos animales (32). Es interesante destacar que en el grupo de animales que recibió una dieta enriquecida con AGPI omega-6, el efecto fue opuesto. Además, fue posible retrasar el crecimiento tumoral en ratones transgénicos FAT-I, los que pueden convertir a los ácidos grasos omega-6 en omega-3, esto es pueden formar EPA y DHA a partir de AL (25).

\section{Modelos de estudio de cáncer de mama (CaM)}

Para el CaM también se ha demostrado el efecto protector de los AGPICL omega-3 $(33,34)$, y es así como un estudio realizado en ratas, demostró que una dieta enriquecida con DHA indujo una reducción de los tumores mamarios, con un aumento de un $60 \%$ en los niveles plasmáticos de la proteína supresora de tumores BRCA1 (35). En los animales que recibieron las dietas enriquecidas con ácidos grasos omega-3 se observó un aumento en la eficacia de los fármacos doxorrubicina y mitomicina $\mathrm{C}$, los cuales se utilizan para inhibir el crecimiento tumoral (36). Además, se observó un aumento del efecto inhibitorio del fármaco tamoxifeno en modelos estrógeno-dependientes (37), lo cual permitiría plantear el potencial uso de los AGPICL omega-3 como co-ayudantes en la quimioterapia estándar para el tratamiento del CaM.

\section{Modelos en cáncer de colon $(\mathrm{CaC})$}

En el caso del CaC los AGPICL omega-3 también han demostrado ejercer un rol protector frente a la enfermedad. Es así como en ratas con $\mathrm{CaC}$ alimentadas con dietas enriquecidas en AGPICL omega-3 se observó una disminución del crecimiento tumoral y una inhibición de la metástasis de las células cancerígenas (38). Un estudio 
demostró que los AGPICL omega-3, al inhibir la actividad de la enzima COX-2, disminuyen el crecimiento tumoral (39). Sin embargo, en otros casos no es posible observar este efecto, lo cual indicaría que existirían isoformas de la COX-2 cuya actividad es independiente de la regulación que ejercen los AGPICL omega- 3 en su funcionamiento (40). Aunque el efecto supresor del crecimiento tumoral es bastante claro cuando se usan ácidos grasos omega-3 de origen marino (EPA y DHA), este efecto resulta más bien controversial cuando se utiliza un ácido graso omega- 3 de origen terrestre (ALN). Aunque varios estudios han informado que el aceite de linaza tendría un efecto anticancerígeno a nivel de colon por su contenido de ALN (41), existe consenso que sólo el EPA y el DHA provocarían una reducción en el volumen tumoral, como se observó en ratones ApcMin, los cuales generan espontáneamente tumores intestinales (42).

La información obtenida de estudios epidemiológicos (23), combinada con los efectos beneficiosos de los AGPICL omega-3 observados en modelos animales $(31,32,35)$ y en cultivos celulares $(38,40)$ en relación al $\mathrm{Ca}$, ha motivado el desarrollo de múltiples intervenciones clínicas usando aceite de pescado rico AGPICL omega-3, en la prevención y el tratamiento de esta enfermedad, así como para el apoyo nutricional de los pacientes con cáncer, con el objetivo de reducir la pérdida de peso y contribuir a restablecer la función del sistema inmune. A continuación se hace referencia a una serie de ensayos clínicos donde se han utilizado ácidos grasos omega-3 en la prevención y el tratamiento de determinados tipos de $\mathrm{Ca}$.

\section{APLICACIONES CLÍNICAS DE LOS AGPICL OMEGA-3 EN PACIENTES CON CÁNCER}

Varios ensayos clínicos han abordado el uso de suplementos nutricionales que contienen AG omega-3 para reducir la pérdida de peso (caquexia) en pacientes con neoplasias malignas avanzadas. Los primeros estudios establecieron que el uso de AGPICL omega-3 permitía estabilizar la pérdida, e incluso lograr un aumento de peso, en pacientes caquéxicos portadores de un cáncer avanzado $(43,44)$. Un problema no menor en el uso de los AGPICL omega-3 es la intolerancia (principalmente gastrointestinal) frente a su administración, siendo un problema crucial a la hora de buscar efectos beneficiosos. Sin embargo, cuando los pacientes son capaces de ingerir y tolerar los suplementos, es posible estabilizar la pérdida de peso e incluso lograr pequeños incrementos de este (45). Esto sugiere que los AGPICL omega-3 pueden tener un papel en el soporte nutricional de pacientes con cáncer, especialmente en $\mathrm{CaP}, \mathrm{CaM}$ y $\mathrm{CaC}$.

\section{AGPICL OMEGA-3 EN LA PREVENCIÓN Y TRATAMIENTO DEL CÁNCER}

Existe evidencia respecto a que los inhibidores de la COX-2, al bloquear el metabolismo del AA, serían una primera estrategia en la prevención del $\mathrm{CaP}$ y $\mathrm{CaC}$ (46). Sin embargo, la toxicidad cardiovascular de los inhibidores de la COX-2 ha puesto en peligro su utilidad clínica (47). Reducir la ingesta de AGPI omega-6 y aumentar la ingesta de AGPI omega-3 en la dieta podría ser una intervención atractiva para reducir la producción de eicosanoides pro-inflamatorios y prevenir el desarrollo del cáncer (48).

Estudios orientados a la prevención del cáncer han demostrado que mediante el uso de AGPICL omega-3 es posible reducir el riesgo de desarrollar $\mathrm{CaC}$, utilizando como indicador la disminución en la proliferación celular en la mucosa colónica o del recto (49). Un estudio aleatorizado, doble ciego, estableció que tras la administración de aceite de pescado durante 30 días a personas con alto riesgo, se observó una disminución en la proliferación celular de la mucosa rectal y en el riesgo de presentar este tipo de cáncer (50). Además, fue posible establecer un aumento en los niveles de EPA y DHA y una disminución de los niveles de AA en la mucosa rectal (51).

En otro estudio aleatorio, doble-ciego, realizado en personas sanas a las cuales se les dio una dieta en la cual se incorporó aceite de pescado o aceite de maíz durante cuatro semanas, se estableció una disminución significativa en la proliferación de las células del recto, menor actividad de la enzima ornitina descarboxilasa y menor liberación de $\mathrm{PGI}_{2}$, en aquellas personas que consumieron aceite de pescado, pero no en aquellas que consumieron aceite de maíz (52). Sin embargo, cuando se repitió el ensayo con una dieta alta en grasas $(50 \%$ de la calorías/día), no se encontraron diferencias significativas, lo que indica que la menor relación AG omega-6/ omega-3 es crucial para determinar los efectos del aceite de pescado sobre el riesgo de desarrollar cáncer $(53,54)$.

El CaP es un segundo tipo de cáncer en el cual se han estudiado los efectos quimioprotectores de los AGPICL omega-3. Estudios preliminares han demostrado que una intervención de 3 meses, en una dieta con un bajo aporte de grasa y utilizando aceite de pescado como fuente de AGPICL omega-3, fue capaz de disminuir la relación de AG omega-6/omega-3, tanto en plasma como en tejido adiposo, lo cual podría indicar una disminución en la generación de moléculas pro inflamatorias y pro cancerígenas en pacientes con $\mathrm{CaP}$, frente a lo cual se hace obviamente necesario realizar más investigaciones (55).

En otro estudio realizado en pacientes de $\mathrm{CaP}$, quienes recibieron una dieta baja en grasas que contenía semillas de lino como fuente de ALN, se produjo 
una disminución en los niveles de antígeno prostático específico y del colesterol total plasmático, así como la proliferación de células epiteliales en muestras de biopsia de inflamación prostática benigna (56). En este sentido, aún están pendientes estudios que busquen establecer las dosis específicas de AG omega-3 y los tiempos de administración de las diferentes fuentes de ácidos grasos a utilizar (de origen terrestre o marino), ya sea precursores (ALN) o preformados (EPA y/o DHA), la interacción con otros nutrientes, los mecanismos y vías de acción, los tejidos más sensibles al tratamiento, entre otros.

\section{CONCLUSIONES}

Los profundos y significativos cambios que ha experimentado la dieta del ser humano, especialmente en occidente desde la revolución industrial hasta nuestros días, han producido una profunda alteración en los procesos metabólicos y en la salud de la población. En relación a los lípidos, la balanza se ha inclinado progresivamente a favor de un consumo creciente de AG omega-6, gatillando así un estado pro-inflamatorio constante. La reestructuración de nuestra alimentación hacia una dieta más saludable y por sobre todo, tendiente a equilibrar el aporte dietario y la relación de consumo de los AGPI omega-6 y omega-3 sería un enfoque atractivo para una adecuada nutrición y eventual quimioprevención del cáncer. En efecto, a diferencia de muchos otros agentes quimioprotectores, los AGPICL omega-3 son nutrientes esenciales, con efectos benéficos adicionales para el ser humano, especialmente a nivel cardiovascular, neurológico, inmunológico, entre otros. Considerando el rol protector que en la actualidad se le puede conferir a los AGPICL omega-3 frente al cáncer, especialmente los discutidos en este artículo, resulta fundamental estimular el consumo de una dieta rica en pescados grasos (salmón, jurel, atún, entre otros) o suplementos alimentarios que contengan AGPICL omega-3 de origen marino.

\section{RESUMEN}

Los ácidos grasos poliinsaturados de cadena larga omega-3 (AGPICL omega-3) son esenciales para el ser humano los que deben ser incorporados al organismo en la dieta. Desde hace casi un siglo se sabe que estos ácidos grasos son fundamentales para el crecimiento y desarrollo del individuo y desde 1970 en adelante, se han encontrado sólidas evidencias para atribuir diversas propiedades saludables a estos ácidos grasos, especialmente a nivel cardiovascular, neurológico y antiinflamatorio. En este sentido, en la actualidad un gran número de investigaciones se han centrado en estudiar el rol protector de los AGPICL omega-3 respecto al cáncer, principalmente por las propiedades antiinflamatorias que poseen. En el presente trabajo se exponen una serie de evidencias que permiten establecer un posible uso preventivo y terapéutico de los AGPICL omega-3 frente al cáncer, específicamente el de próstata, mama y colon.

Palabras clave: cáncer, ácidos grasos, ácidos grasos omega-3, efecto antiinflamatorio.

Dirigir la correspondencia a:

Profesor

Rodrigo Valenzuela B.

Escuela de Nutrición y Dietética

Facultad de Medicina

Universidad de Chile

Independencia 1027

Casilla 1227. Independencia,

Santiago, Chile.

Teléfonos: 56-2-9786746; 56-2-9786085; 56-9-78098733

E-mail: rvalenzuelab@med.uchile.c1

\section{BIBLIOGRAFÍA}

1. GuptaA, Gupta V. Metabolic syndrome: What are the risks for humans?. Biosci Trends. 2010;4(5):204-12.

2. Varela-Moreiras G, Avila JM, Cuadrado C, del Pozo S, Ruiz E, Moreiras O. Evaluation of food consumption and dietary patterns in Spain by the Food Consumption Survey: updated information. Eur J Clin Nutr. 2010; 64 Suppl 3:S37-43.

3. Carlson B, Kingston J. Docosahexaenoic acid, the aquatic diet, and hominid encephalization: difficulties in establishing evolutionary links. Am J Hum Biol. 2007; 19(1):132-41.

4. Simopoulos A. Essential fatty acids in health and chronic diseases. Am J Clin Nutr. 2003; 56:67-70.

5. Simopoulos A. The importance of the omega-6/ omega-3 fatty acid ratio in cardiovascular disease and other chronic diseases. Exp Biol Med 2008; 233:674-88.

6. Martinelli N, Consoli L, Olivieri O. A 'desaturase hypothesis' for atherosclerosis: Janus-faced enzymes in omega- 6 and omega- 3 polyunsaturated fatty acid metabolism. J Nutrigenet Nutrigenomics. 2009; 2:129-39.

7. He K. Fish, long-chain omega-3 polyunsaturated fatty acids and prevention of cardiovascular disease-eat fish or take fish oil supplement? Prog Cardiovasc Dis. 2009; 52:95-114.

8. Sharma M., Ansari M, Abou-Setta A, Soares-Weiser K, Ooi T, Sears M, Yazdi F, Tsertsvadze A, Moher D. Systematic review: comparative effectiveness 
and harms of combination therapy and monotherapy for dyslipidemia. Ann Intern Med. 2009; (3) 151:622-30.

9. Houston M, Fazio S, Chilton F, Wise D, Jones K, Barringer T, Bramlet D. Nonpharmacologic treatment of dyslipidemia. Prog Cardiovasc Dis. 2009; 52:61-94.

10. Burr G, Burr M. On the nature and role of fatty acids essential in nutrition. J Biol Chem 1930; 86:587-621.

11. Holman R. The slow discovery of the importance of omega 3 essential fatty acids in human health. $\mathrm{J}$ Nutr 1998; 128:427S-33S.

12. Tvrdik P, Westerberg R, Silve S, Asadi A, Jakobsson A, Cannon B, Loison G, Jacobsson A. Role of a new mammalian gene family in the biosynthesis of very long chain fatty acids and sphingolipids. J Cell Biol 2000; 149:707-18.

13. Igarashi M, DeMar Jr J, Ma K, Chang L, Bell J, Rapoport S. Docosahexaenoic acid synthesis from alpha-linolenic acid by rat brain is unaffected by dietary n-3 PUFA deprivation. J Lipid Res 2007; 48:1150-8.

14. Igarashi M, DeMar Jr J, Ma K, Chang L, Bell J, Rapoport S. Upregulated liver conversion of alphalinolenic acid to docosahexaenoic acid in rats on a 15 week n-3 PUFA-deficient diet. J Lipid Res 2007; 48:152-64.

15. Sargent J. Fish Oils and Human Diet. Br J Nutr 1997; 78: S5-S13

16. Crawford M. The role of dietary fatty acid in biology: their place in the evolution of the human brain. Nutr Rev 1992; 503-11.

17. Rapoport S, Rao J, Igarashi M. Brain metabolism of nutritionally essential polyunsaturated fatty acids depends on both the diet and the liver. Prostag Leukotr Essent Fatty Acids 2007; 77:251-61.

18. Kimura Y, Kono S, Toyomura K, Nagano J, Mozoue T, Moore M, Mibu R, Tanaka M, Kakeji Y, Maehara Y, Okamura T, Ikejiri K, Futami K, Yasunami Y, Maekawa T, Takenaka K, Ichimiya H, Imaizumi N. Meat fish and fat intake in relation to subside specific risk of colorectal cancer: The Fukuoka Colorectal Cancer Study. Cancer Sci 2007; 98: 590-7.

19. Theodoratou E, Mc Neill G, Cetnarskyj R, Farrington $\mathrm{S}$, Tenesa A. Dietary fatty acids and colorectal cancer: a case-control study. Am J Epidemiol 2007; 166:181-95.

20. Habermann N, Christian B, Luckas B, Pool-Zobel B, Lund E, Glei M. Effects of fatty acids on metabolism and cell growth of human colon cell lines of different transformation state. Biofactors. 2009; 35(5):460-7.

21. Chavarro J, Stampfer M, Li H, Campos H, Kurth T, Ma J. A prospective study of polyunsaturated fatty acid levels in blood and prostate cancer risk. Cancer Epidemiol Biomarkers Prev. 2007; 16: 1364-70.

22. Ritch C, Wan R, Stephens L, Taxy J, Huo D, Gong E, Zagaja G, Brendler C. Dietary fatty acid correlated with prostate cancer biopsy grade and volume in Jamaican men. J Urol. 2007; 177:97-101.

23. Reese A, Fradet V, Witte J. Omega-3 fatty acids, genetic variants in COX-2 and prostate cancer. $\mathrm{J}$ Nutrigenet Nutrigenomics. 2009; 2(3):149-58.

24. Habermann N, Lund E, Pool-Zobel B, Glei M. Modulation of gene expression in eicosapentaenoic acid and docosahexaenoic acid treated human colon adenoma cells. Genes Nutr 2009; 4(1):73-6

25. Berquin I, Min Y, Wu R, Wu J, Perry D, Cline J, Thomas M, Thornburg T, Kulik G, Smith A. Modulation of prostate cancer genetic risk by omega-3 and omega- 6 fatty acids. J Clin Invest 2007; 117:1866-75.

26. Van Beelen V, Roeleveld J, Mooibroek H, Sijtsma L, Bino R, Bosch D, Rietjens I, Alink G. A comparative study on the effect of algal and fish oil on viability and cell proliferation of Caco-2 cells. Food Chem Toxicol 2007; 45(5):716-24.

27. Chapkin R, Seo J, McMurray D, Lupton J. Mechanisms by which docosahexaenoic acid and related fatty acids reduce colon cancer risk and inflammatory disorders of the intestine. Chem Phys Lipids 2008; 153(1):14-23.

28. Siezen C, Van Leeuwen A, Kram N, Luken M, Van Kranen H, Kampman E. Colorectal adenoma risk is modified by the interplay between polymorphisms in arachidonic acid pathway genes and fish consumption. Carcinogenesis 2005; 26: 449-57.

29. Hedelin M, Chang E, Wiklund F, Bellocco R, Klint A, Adolfsson J, Shaedi K, Xu J, Adami H, Gronberg H. Association of frequent consumption of fatty fish whit prostate cancer risk is modified by COX-2 polymorphism. Int J Cancer 2007; 120: 398-405.

30. David D and Cohen L. Effects of dietary menhaden oil and retinyl acetate on the growth of DU 145 human prostatic adenocarcinoma cells transplanted into athymic nude mice. Carcinogenesis 1988; 9: 603-5.

31. Kobayashi N, Barnard R, Henning S, Elashoff D, Reddy S, Cohen P, Leung P, Hong-Gonzalez J, Freedland SJ, Said J, Gui D, Seeram N, Popoviciu LM, Bagga D, Heber D, Glaspy J, Aronson W. Effect of altering dietary omega-6/omega-3 fatty acid 
ratios on prostate cancer membrane composition, cyclooxygenase-2, and prostaglandin E2. Clin. Cancer Res 2006; 12:4662-70.

32. Wang S, Gao J, Lei Q, Rozengurt N, Pritchard C, Jiao J, Li G, Roy-Burman P, Nelson P, Liu X, Wu H. Prostate-specific deletion of the murine Pten tumor suppressor gene leads to metastatic prostate cancer. Cancer Cell 2003; 4:209-21.

33. Rose D and Connolly J. Effects of dietary omega-3 fatty acids on human breast cancer growth and metastases in nude mice. J Natl Cancer Inst. 1993; 85:1743-7.

34. Menéndez, A. Vázquez-Martín, S. Ropero, R. Colomer, R. Lupu. HER2 (erbB-2)-targeted effects of the omega-3 polyunsaturated fatty acid, alphalinolenic acid (ALA; 18:3n-3), in breast cancer cells: the "fat features" of the "Mediterranean diet" as an "anti-HER2 cocktail". Clin Transl Oncol 2006; 8:812-20.

35. Jourdan M, Mahéo K, Barascu A, Goupille C, De Latour M, Bougnoux P, Rio P. Increased BRCA1 protein in mammary tumors of rats fed marine omega-3 fatty acids. Oncol Rep 2007; 17:713-19.

36. Hardman W, Avula C, Fernández G, Cameron I. Three percent dietary fish oil concentrate increased efficacy of doxorubicin against MDA-MB 231 breast cancer xenografts. Clin Cancer Res 2001; 7:2041-49.

37. Chen J, Hui E, Ip T, Thompson L. Dietary Flaxseed Enhances the Inhibitory Effect of Tamoxifen on the Growth of Estrogen-Dependent Human Breast Cancer (MCF-7) in Nude Mice. Clin Cancer Res 2004; 10:7703-11.

38. Paulsen J, Elvsaas J, Steffensen I, Alexander J. A fish oil derived concentrate enriched in eicosapentaenoic and docosahexaenoic acid as ethyl ester suppresses the formation and growth of intestinal polyps in the Min mouse. Carcinogenesis 1997; 18:1905-10.

39. Calviello G, Di Nicuolo F, Gragnoli S, Piccioni E. n-3 PUFAs reduce VEGF expression in human colon cancer cells modulating the COX-2/PGE2 induced ERK-1 and - 2 and HIF- $1 \alpha$ induction pathway. Carcinogenesis 2004; 25:2303-10.

40. Reddy B and Maruyama H. Effect of dietary fish oil on azoxymethane-induced colon carcinogenesis in male F344 rats. Cancer Res 1986; 46: 3367-70.

41. Williams D, Verghese M, Walker L, Boateng J, Shackelford L, Chawan C. Flax seed oil and flax seed meal reduce the formation of aberrant crypt foci (ACF) in azoxymethane-induced colon cancer in fisher 344 male rats. Food Chem Toxicol 2007; 45:153-59.
42. Petrik M, McEntee M, Johnson B, Obukowicz M, Whelan J. Highly unsaturated (n-3) fatty acids, but not (alpha)-linolenic, conjugated linoleic or (gamma)-linolenic acids, reduce tumorigenesis in Apc(Min/+) mice. J Nutr 2000; 130:2434-43.

43. Jatoi A, Rowland K, Loprinzi C, Sloan J, Dakhil S, MacDonald N, Gagnon B, Novotny P, Mailliard J, Bushey T, Nair S, Christensen B; North Central Cancer Treatment Group. An eicosapentaenoic acid supplement versus megestrol acetate versus both for patients with cancer-associated wasting: a North Central Cancer Treatment Group and National Cancer Institute of Canada collaborative effort. J Clin Oncol 2004; 22:2469-76.

44. Mantovani G, Macciò A, Madeddu C, Gramignano G, Lusso M, Serpe R, Massa E, Astara G, Deiana L. A phase II study with antioxidants, both in the diet and supplemented, pharmaconutritional support, progestagen, and anti-cyclooxygenase- 2 showing efficacy and safety in patients with cancer-related anorexia/cachexia and oxidative stress. Cancer Epidemiol Biomarkers Prev 2006; 15:1030-34.

45. Dewey A, Baughan C, Dean T, Higgins B, Johnson I. Eicosapentaenoic acid (EPA, an omega-3 fatty acid from fish oils) for the treatment of cancer cachexia. Cochrane Database Syst Rev 2007; 24:CD004597.

46. Szymczak M, Murray M, Petrovic N. Modulation of angiogenesis by omega-3 polyunsaturated fatty acids is mediated by cyclooxygenases. Blood 2008; 111(7):3514-21.

47. Reese A, Fradet V, Witte J. Omega-3 fatty acids, genetic variants in COX-2 and prostate cancer. $\mathrm{J}$ Nutrigenet Nutrigenomics 2009; 2:149-58.

48. Varma S, Eskin M, Bird R, Dolenko B, Raju J, Ijare $\mathrm{O}$, Bezabeh T. Potential of magnetic resonance spectroscopy in assessing the effect of fatty acids on inflammatory bowel disease in an animal model. Lipids 2010; 45(9):843-54.

49. Ramalho T, Rocha M., da Cunha E, Freitas M. The search for new COX-2 inhibitors: a review of 2002 - 2008 patents. Expert Opinion on Therapeutic Patents 2009; 19:1193-228.

50. Hofmanová J, Vaculová A, Koubková Z, Hýzd'alová M, Kozubík A. Human fetal colon cells and colon cancer cells respond differently to butyrate and PUFAs. Mol Nutr Food Res 2009; 53 Suppl 1:S102-13.

51. Solomon S, McMurray J, Pfeffer M, Wittes J, Fowler R, Finn P, Anderson W, Zauber A, Hawk E, Bertagnolli M; Adenoma prevention with Celecoxib (APC). Study investigators. Cardiovascular risk associated with Celecoxib in a clinical trial for colorectal adenoma prevention. Engl J Med 2005; 
352:1071-80.

52. Yi Fan Y, Spencer T, Wang N, Moyer M, Chapkin S. Chemopreventive n-3 fatty acids activate RXR $\alpha$ in colonocytes. Carcinogenesis 2003; 24: 1541-8.

53. Bartram H, Gostner A, Scheppach W, Reddy S, Rao C, Dusel G, Richter F, Richter A, Kasper H. Effects of fish oil on rectal cell proliferation, mucosal fatty acids, and prostaglandin E2 release in healthy subjects. Gastroenterol 1993; 105:1317-22.

54. Bartram H, Gostner A, Reddy B, Rao C, Scheppach W, Dusel G, Richter A, Kasper H. Missing antiproliferative effect of fish oil on rectal epithelium in healthy volunteers consuming a high-fat diet: potential role of the n-3: n-6 fatty acid ratio. Eur J Cancer Prev 1995; 4:231-7.

55. Aronson W, Glaspy J, Reddy S, Reese D, Heber D, Bagga D. Modulation of omega-3/omega- 6 polyunsaturated ratios with dietary fish oils in men with prostate cancer. Urology 2001; 58:283-8.

56. Demark-Wahnefried W, Robertson C, Walther P, Polascik T, Paulson D, Vollmer R. Pilot study to explore effects of low-fat, flaxseed-supplemented diet on proliferation of benign prostatic. Urology 2004; 63:900-4. 